\begin{tabular}{|c|c|}
\hline & $\begin{array}{l}\text { International Journal of Trend in Scientific } \\
\text { Research and Development (IJTSRD) }\end{array}$ \\
\hline 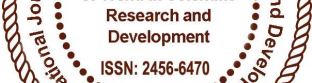 & International Open Access Journal \\
\hline 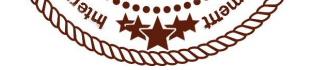 & ISSN No: 2456 - 6470 | www.ijtsrd.com | Volume - 2 | Issue - 2 \\
\hline
\end{tabular}

\title{
Some new sets and a new decomposition of fuzzycontinuity, fuzzy almost strongI-continuity via idealization
}

\begin{abstract}
K. Malarvizhi
Assistant Professor, Department of Mathematics, Sri Krishna Arts and Science College, Kuniamuthur, Coimbatore, Tamil Nadu, India
\end{abstract}

\author{
R. SriKirithiga \\ PG Student, Department of \\ Mathematics, Sri Krishna Arts and \\ Science College, Kuniamuthur, \\ Coimbatore, Tamil Nadu, India
}

\begin{abstract}
N. Arulselvam
PG Student, Department of Mathematics, Sri Krishna Arts and Science College, Kuniamuthur, Coimbatore, Tamil Nadu, India
\end{abstract}

\section{ABSTRACT}

In this paper, we discuss the notion of fuzzy strong $\beta$ I -open sets. We introduce fuzzy almost I -open, fuzzy almost strong I -open sets, fuzzy S $\beta I$-set, fuzzy DI sets closely related with fuzzy strong $\beta-\mathrm{I}$-open sets. Additionally, we investigate some characterizations and properties of these sets. With the help of fuzzy strong $\beta$-I -open set and fuzzy DI -set to obtain a new decomposition of fuzzy continuity viaidealization.

Keywords: Fuzzy topological ideal; fuzzy strong $\beta-I$ open set; fuzzy DI -set; fuzzy SBI -set; fuzzy almost I open; fuzzy almost strong I -open set

\section{Introduction and Preliminaries}

Fuzziness is one of the most important and useful concepts in the modern scientific studies. In 1965, Zadeh[11] first introduced the notion of fuzzy sets. In 1945, Vaidyanathaswamy[9] introduced the concepts of ideal topological spaces. In 1990, Jankovic and Hamlett[4] have defined the concept of I -open set via localfunction in ideal topological space. In 1997, Mahmoud[6] and sarkar[8] independently presented some of the ideal concepts in the fuzzy trend and studied many other properties. Decomposition of fuzzy continuity is one of the many problems in the fuzzy topology. It becomes very interesting when decomposition is done via fuzzytopological ideals.

Throughout this paper, $\mathrm{X}$ represents a nonempty fuzzy set and fuzzy subset $\mathrm{A}$ of $\mathrm{X}$, denoted by $\mathrm{A} \leq \mathrm{X}$, is characterized by a membership function in the sense of Zadeh[11]. The basic fuzzy sets are the empty set, the whole set and the class of all fuzzy subsets of $\mathrm{X}$ which will be denoted by 0,1 and $\mathrm{I}^{\mathrm{X}}$ respectively. A subfamily $\tau$ of $\mathrm{I}^{\mathrm{X}}$ will denote topology of fuzzy sets on $\mathrm{I}^{\mathrm{X}}$ as defined by Chang[3]. By $(\mathrm{X}, \tau)$, we mean a fuzzy topological space in Chang's sense. A fuzzy point in $X$ with support $\mathrm{x} \in \mathrm{X}$ and value $\alpha(0<\alpha \leq 1)$ is denoted by $\mathrm{x}_{\alpha}$. For a fuzzy subset $\mathrm{A}$ of $\mathrm{X}, \mathrm{Cl}(\mathrm{A}), \operatorname{Int}(\mathrm{A})$ and $1-$ Awill respectively, denote the fuzzy closure, fuzzy interior and fuzzy complement of A. A nonempty collection I of fuzzy subsets of X is called a fuzzyideal[8] if and only if

1. $\mathrm{B} \in \mathrm{I}$ and $\mathrm{A} \leq \mathrm{B}$, then $\mathrm{A} \in \mathrm{I}$ (heredity),

2. if $A \in I$ and $B \in I$ then $A \vee B \in I$ (finite additivity). 


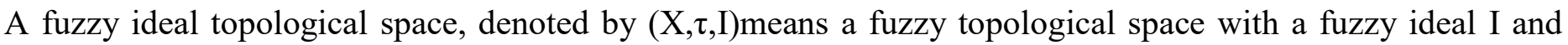
fuzzy topology $\tau$. For $(\mathrm{X}, \tau, \mathrm{I})$, the fuzzy local function of $\mathrm{A} \leq \mathrm{X}$ with respect to $\tau$ and $\mathrm{I}$ is denoted by $\mathrm{A}^{*}(\tau, \mathrm{I})\left(\right.$ briefly $\left.\mathrm{A}^{*}\right)$ and is defined as $\mathrm{A}^{*}(\tau, \mathrm{I})=\mathrm{V}\{\mathrm{x} \in \mathrm{X}: \mathrm{A} \wedge \mathrm{U} / \in \mathrm{I}$ for every $\mathrm{U} \in \tau(\mathrm{x})\}$. While $\mathrm{A}^{*}$ is the union of the fuzzy points $x$ such that if $U \in \tau(x)$ and $E \in I$, then there is at least one $y \in X$ for which $U(y)+A(y)-1>$ $\mathrm{E}(\mathrm{y})$. Fuzzy closure operator of a fuzzy set in $(\mathrm{X}, \tau, \mathrm{I})$ is defined as $\mathrm{Cl}^{*}(\mathrm{~A})=\mathrm{A} \vee \mathrm{A}^{*} \cdot \operatorname{In}(\mathrm{X}, \tau, \mathrm{I})$, the collection $\tau^{*}(\mathrm{I})$ means an extension of fuzzy topological space than $\tau$ via fuzzy ideal which is constructed by considering the class

$\beta=\{U-E: U \in \tau, \in I\}$ as a base[8]. This topology of fuzzy sets is considered as generalization of the ordinary one.

First, we shall recall some definitions used in the sequel.

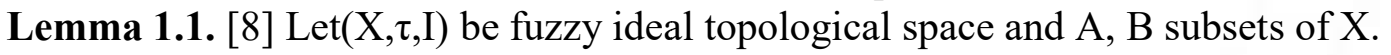

The following properties hold:

(a) If $\mathrm{A} \leq \mathrm{B}$, then $\mathrm{A}^{*} \leq \mathrm{B}^{*}$,

(b) $(\mathrm{A} \vee \mathrm{B})^{*}=\mathrm{A}^{*} \vee \mathrm{B}^{*}$,

(c) $\mathrm{A}^{*}=\mathrm{Cl}\left(\mathrm{A}^{*}\right) \leq \mathrm{Cl}(\mathrm{A})$,

(d) if $U \in \tau$, then $U \wedge A^{*} \leq(\mathrm{U} \wedge \mathrm{A})^{*}$,

(e) if $\mathrm{U} \in \tau$, then $\mathrm{U} \wedge \mathrm{Cl}^{*}(\mathrm{~A}) \leq \mathrm{Cl}^{*}(\mathrm{U} \wedge \mathrm{A})$.

Lemma 1.2. [5] Let $(X, \tau)$ be an ideal topological space with an arbitrary index $\Delta, I$ an ideal of subsets of $X$ and $\rho(X)$ the power set of $X$. If $\left\{A_{\alpha}: \alpha \in \Delta\right\} \leq \rho(X)$, then the following property holds:

$$
\vee_{\alpha \in \Delta}\left(A_{\alpha}^{*}\right) \leq\left(\vee_{\alpha \in \Delta} A_{\alpha}\right)^{*}
$$

Definition 1.1. A subset A of a space $(X, \tau, I)$ is said to be

(a) fuzzy $\alpha-\mathrm{I}-$ open[10] if $\mathrm{A} \leq \operatorname{Int}\left(\mathrm{Cl}^{*}(\operatorname{Int}(\mathrm{A}))\right)$,

(b) fuzzy $\beta-\mathrm{I}$-open[10] if $\mathrm{A} \leq \mathrm{Cl}\left(\operatorname{Int}\left(\mathrm{Cl}^{*}(\mathrm{~A})\right)\right)$.

(c) fuzzy I [2]-open if $\mathrm{A} \leq \operatorname{Int}\left(\mathrm{A}^{*}\right)$.

(d) fuzzy pre-I -open[2] if $\mathrm{A} \leq \operatorname{Int}\left(\mathrm{Cl}^{*}(\mathrm{~A})\right)$.

(e) fuzzy semi-I -open[1] if $\mathrm{A} \leq \mathrm{Cl}^{*}(\operatorname{Int}(\mathrm{A}))$.

Definition 1.2. [10] A subset $A$ of a space $(X, \tau)$ is said to be fuzzy $\beta$-open if

$\mathrm{A} \leq \mathrm{Cl}(\operatorname{Int}(\mathrm{Cl}(\mathrm{A})))$.

Definition 1.3. [12] A subset $A$ of a space $(X, \tau, I)$ is said to be fuzzy $*-I$-dense in itself if $A \leq A^{*}$.

\section{Fuzzy Strong $\beta$-I-open set and Almost Fuzzy Strong I-open set}

Definition 2.1. A subset $A$ of a space $(X, \tau, I)$ is said to be fuzzy almost -I -open if $A \leq C l\left(\operatorname{Int}\left(A^{*}\right)\right)$.

Definition 2.2. A subset $A$ of a space(X, $\tau$,I)is said to be fuzzy strong $\beta$-I -open if $\mathrm{A} \leq \mathrm{Cl}^{*}\left(\operatorname{Int}\left(\mathrm{Cl}^{*}(\mathrm{~A})\right)\right)$. We denote the family of all fuzzy strong $\beta$-I -open set of $(\mathrm{X}, \tau, \mathrm{I})$ by $\mathrm{Fs} \beta \mathrm{IO}(\mathrm{X})$.

Definition 2.3. A subset A of a fuzzy ideal topological space(X, $\tau, \mathrm{I})$ is said to be almost fuzzy strong-I -open if $\mathrm{A} \leq \mathrm{Cl}^{*}\left(\operatorname{Int}\left(\mathrm{A}^{*}\right)\right)$. We denote the family of all fuzzy almost strong-I -open set of $(\mathrm{X}, \tau, \mathrm{I})$ by FasIO(X).

Proposition 2.1. For a subset of a fuzzy ideal topological space $(\mathrm{X}, \tau, \mathrm{I})$ the followinghold: 
(a) Every fuzzy semi-I -open set is fuzzy strong $\beta$-I -open.

(b) Every fuzzy $\beta$-I -open is fuzzy $\beta$-open.

Proof: (a) Let A be a fuzzy semi-I -open set. Then $\mathrm{A} \leq \mathrm{Cl}^{*}(\operatorname{Int}(\mathrm{A})) \leq \mathrm{Cl}^{*}\left(\operatorname{Int}\left(\mathrm{Cl}^{*}(\mathrm{~A})\right)\right)$.

This shows that $A \in \operatorname{Fs} \beta I O(X)$.

(b) Let $\mathrm{A}$ is fuzzy $\beta$-I -open. We have, $\mathrm{A} \leq \mathrm{Cl}(\operatorname{Int}(\mathrm{Cl}(\mathrm{A}))) \leq \mathrm{Cl}(\operatorname{Int}(\mathrm{Cl}(\mathrm{A})))$. Therefore, $\mathrm{A}$ is fuzzy $\beta$-open.

Remark 2.1. The converses of each statement in proposition 2.1 need not be true as the following examples show.

Example 2.1. Let $X=\{a, b, c\}$ and $A, B, C$ be fuzzy subsets of $X$ defined asfollows:

$$
\begin{aligned}
& \mathrm{A}(\mathrm{a})=0.3, \mathrm{~A}(\mathrm{~b})=0.2, \mathrm{~A}(\mathrm{c})=0.4 \\
& \mathrm{~B}(\mathrm{a})=0.8, \mathrm{~B}(\mathrm{~b})=0.8, \mathrm{~B}(\mathrm{c})=0.4
\end{aligned}
$$

Let $\tau=\{0, \mathrm{~B}, 1\}$. If we take $\mathrm{I}=\{0\}$, then A is fuzzy strong $\beta$-I -open but A isnot fuzzy semi-I -open set.

Example 2.2. Let $X=\{a, b, c\}$ and $A, B, C$ be fuzzy subsets of $X$ defined asfollows:

$$
\begin{aligned}
& \mathrm{A}(\mathrm{a})=0.2, \mathrm{~A}(\mathrm{~b})=0.4, \mathrm{~A}(\mathrm{c})=0.7 \\
& \mathrm{~B}(\mathrm{a})=0.2, \mathrm{~B}(\mathrm{~b})=0.6, \mathrm{~B}(\mathrm{c})=0.7
\end{aligned}
$$

Let $\tau=\{0, B, 1\}$. If we take $I=\rho(X)$, then $A$ is fuzzy $\beta$-open but $A$ is not fuzzy $\beta$-I -open set.

Proposition 2.2. For a subset of a fuzzy ideal topological space $(X, \tau, I)$ the followinghold:

(a) Every fuzzy I -open set is fuzzy almost strong-I -open.

(b) Every fuzzy almost strong-I -open is fuzzy strong $\beta$-I -open.

Proof :The proof is similar with the that Proposition 2.1.

Remark 2.2. The converses of each statement in proposition 2.2 need not be true as the following examples show.

Example 2.3. Let $X=\{a, b, c\}$ and $A, B$ be fuzzy subsets of $X$ defined as follows:

$$
\begin{aligned}
& \mathrm{A}(\mathrm{a})=0.2, \mathrm{~A}(\mathrm{~b})=0.3, \mathrm{~A}(\mathrm{c})=0.7 \\
& \mathrm{~B}(\mathrm{a})=0.1, \mathrm{~B}(\mathrm{~b})=0.2, \mathrm{~B}(\mathrm{c})=0.2
\end{aligned}
$$

Let $\tau=\{0, B, 1\}$. If we take $\mathrm{I}=\{0\}$, then A is fuzzy almost strong-I -open but A is not fuzzy I -open set.

Example 2.4. Let $X=\{a, b, c\}$ and $A, B$ be fuzzy subsets of $X$ defined as follows:

$$
\begin{aligned}
& \mathrm{A}(\mathrm{a})=0.5, \mathrm{~A}(\mathrm{~b})=0.4, \mathrm{~A}(\mathrm{c})=0.6 \\
& \mathrm{~B}(\mathrm{a})=0.5, \mathrm{~B}(\mathrm{~b})=0.5, \mathrm{~B}(\mathrm{c})=0.6
\end{aligned}
$$

Let $\tau=\{0, \mathrm{~A}, 1\}$. If we take $\mathrm{I}=\rho(\mathrm{X})$, then $\mathrm{A}$ is fuzzy strong- $\beta$-I -open but A isnot fuzzy almost strong-I -open set.

Proposition 2.3. Let $(\mathrm{X}, \tau, \mathrm{I})$ be a fuzzy ideal topological space with an arbitrary index set $\Delta$. Then the following properties hold:

(a) if $\left\{\mathrm{A}_{\alpha}: \alpha \in \Delta\right\} \leq \mathrm{Fs} \beta \mathrm{IO}(\mathrm{X})$, then $\mathrm{V}\left\{\mathrm{A}_{\alpha}: \alpha \in \Delta\right\} \in \mathrm{Fs} \beta \mathrm{IO}(\mathrm{X})$.

(b) if $A \in \operatorname{Fs} \beta I O(X)$ and $U \in \tau$, then $(U \wedge V) \in \operatorname{Fs} \beta I O(X)$. 


\section{Proof :}

(a) Since $\left\{\mathrm{A}_{\alpha}: \alpha \in \Delta\right\} \leq \mathrm{Fs} \beta \mathrm{IO}(\mathrm{X}), \mathrm{A}_{\alpha} \leq \mathrm{Cl}^{*}\left(\operatorname{Int}\left(\mathrm{Cl}^{*}\left(\mathrm{~A}_{\alpha}\right)\right)\right)$ for every $\alpha \in \Delta$.

Thus by using Lemma1.1,1.2, we have

$$
\begin{aligned}
\mathrm{V}_{\alpha} \in_{\Delta} \mathrm{A}_{\alpha} \leq \mathrm{V}_{\alpha} \in_{\Delta} \mathrm{Cl}^{*}\left(\operatorname{Int}\left(\mathrm{Cl}^{*}\left(\mathrm{~A}_{\alpha}\right)\right)\right)=\mathrm{V}_{\alpha} \in_{\Delta}\left[\operatorname{Int}\left(\mathrm{Cl}^{*}\left(\mathrm{~A}_{\alpha}\right)\right) \vee\left(\operatorname{Int}\left(\mathrm{Cl}^{*}\left(\mathrm{~A}_{\alpha}\right)\right)\right)^{*}\right] \\
=\left[\mathrm{V}_{\alpha} \in_{\Delta}\left(\operatorname{Int}\left(\mathrm{Cl}^{*}\left(\mathrm{~A}_{\alpha}\right)\right)\right)\right] \vee\left[\mathrm{V}_{\alpha} \in_{\Delta}\left(\operatorname{Int}\left(\mathrm{Cl}^{*}\left(\mathrm{~A}_{\alpha}\right)\right)\right)^{*}\right] \\
\leq\left[\operatorname{Int}\left(\mathrm{V}_{\alpha} \in_{\Delta}\left(\mathrm{Cl}^{*}\left(\mathrm{~A}_{\alpha}\right)\right)\right)\right] \vee\left[\operatorname{Int}\left(\mathrm{V}_{\alpha} \in_{\Delta}\left(\mathrm{Cl}^{*}\left(\mathrm{~A}_{\alpha}\right)\right)\right)\right]^{*} \\
=\left(\left[\operatorname{Int}\left(\mathrm{V}_{\alpha} \in_{\Delta}\left(\mathrm{A}_{\alpha} \vee \mathrm{A}_{\alpha}^{*}\right)\right)\right] \vee\left[\operatorname{Int}\left(\mathrm{V}_{\alpha} \in_{\Delta}\left(\mathrm{A}_{\alpha} \vee \mathrm{A}_{\alpha}^{*}\right)\right)\right]^{*}\right) \\
\leq\left[\operatorname{Int}\left(\left(\mathrm{V}_{\alpha} \in_{\Delta} \mathrm{A}_{\alpha}\right) \vee\left(\mathrm{V}_{\alpha} \in_{\Delta} \mathrm{A}_{\alpha}\right)^{*}\right)\right] \vee\left[\operatorname{Int}\left(\left(\mathrm{V}_{\alpha} \in_{\Delta} \mathrm{A}_{\alpha}\right) \vee\left(\mathrm{V}_{\alpha} \in_{\Delta} \mathrm{A}_{\alpha}\right)^{*}\right)\right]^{*} \\
=\operatorname{Int}\left(\mathrm{Cl}^{*}\left(\mathrm{~V}_{\alpha} \in_{\Delta} \mathrm{A}_{\alpha}\right)\right) \vee\left(\operatorname{Int}\left(\mathrm{Cl}^{*}\left(\mathrm{~V}_{\alpha} \in_{\Delta} \mathrm{A}_{\alpha}\right)\right)\right)^{*} \\
=\mathrm{Cl}^{*}\left(\operatorname{Int}\left(\mathrm{Cl}^{*}\left(\mathrm{~V}_{\alpha} \in_{\Delta} \mathrm{A}_{\alpha}\right)\right)\right) .
\end{aligned}
$$

(b) By the hypothesis, $\mathrm{A} \leq \mathrm{Cl}^{*}\left(\operatorname{Int}\left(\mathrm{Cl}^{*}(\mathrm{~A})\right)\right)$ and $\mathrm{U} \leq \operatorname{Int}(\mathrm{U})$. Thus

$(\mathrm{A} \wedge \mathrm{U}) \leq \mathrm{Cl}^{*}\left(\operatorname{Int}\left(\mathrm{Cl}^{*}(\mathrm{~A})\right)\right) \wedge \operatorname{Int}(\mathrm{U})$

$$
\begin{aligned}
& =\left[\operatorname{Int}\left(\mathrm{Cl}^{*}(\mathrm{~A})\right) \vee\left(\operatorname{Int}\left(\mathrm{Cl}^{*}(\mathrm{~A})\right)\right)^{*}\right] \wedge \operatorname{Int}(\mathrm{U}) \\
& =\left[\operatorname{Int}\left(\mathrm{Cl}^{*}(\mathrm{~A})\right) \wedge \operatorname{Int}(\mathrm{U})\right] \vee\left[(\operatorname{Int}(\mathrm{Cl}(\mathrm{A})))^{*} \wedge \operatorname{Int}(\mathrm{U})\right] \\
& \left.\leq\left(\left[\operatorname{Int}\left(\mathrm{Cl}^{*} \mathrm{~A}\right) \wedge \mathrm{U}\right)\right] \vee\left[\left(\operatorname{Int}\left(\mathrm{Cl}^{*}(\mathrm{~A})\right)\right) \wedge \operatorname{Int}(\mathrm{U})\right]^{*}\right) \mid \mathrm{S} \mathrm{Cm} \\
& \leq\left(\left[\operatorname{Int}\left(\left(\mathrm{A} \vee \mathrm{A}^{*}\right) \wedge \mathrm{U}\right)\right] \vee\left[\operatorname{Int}\left(\left(\mathrm{A} \vee \mathrm{A}^{*}\right) \wedge \mathrm{U}\right)\right]^{*}\right) 2456-6 \\
& \leq \operatorname{Int}\left((\mathrm{A} \wedge \mathrm{U}) \vee(\mathrm{A} \wedge \mathrm{U})^{*}\right) \vee\left[\operatorname{Int}\left((\mathrm{A} \wedge \mathrm{U}) \vee(\mathrm{A} \wedge \mathrm{U})^{*}\right)\right]^{*} \\
& =\operatorname{Int}(\mathrm{Cl}(\mathrm{A} \wedge \mathrm{U})) \vee[\operatorname{Int}(\mathrm{Cl} *(\mathrm{~A} \wedge \mathrm{U}))]^{*} \\
& =\mathrm{Cl}(\operatorname{Int}(\mathrm{Cl} *(\mathrm{~A} \wedge \mathrm{U}))) . \operatorname{Thus} \mathrm{A} \wedge \mathrm{U} \in \mathrm{Fs} \beta \operatorname{IO}(\mathrm{X}) .
\end{aligned}
$$

Proposition 2.4. Let $(\mathrm{X}, \tau, \mathrm{I})$ be a fuzzy ideal topological space with an arbitrary index $\Delta$.

Then the following properties hold:

(a) if $\left\{\mathrm{A}_{\alpha}: \alpha \in \Delta\right\} \leq \mathrm{FasIO}(\mathrm{X})$, then $\mathrm{V}\left\{\mathrm{A}_{\alpha}: \alpha \in \Delta\right\} \in \mathrm{FasIO}(\mathrm{X})$.

(b) if $A \in \operatorname{FasiO}(X)$ and $U \in \tau$, then $(U \wedge V) \in \operatorname{FasiO}(X)$.

Proof : The proof is similar with Proposition 2.3.

Proposition 2.5. Let $(X, \tau, I)$ be a fuzzy ideal topological space and $N$ be the ideal of nowhere dense sets of $X$. Then the following properties hold: 
International Journal of Trend in Scientific Research and Development (IJTSRD) ISSN: 2456-6470

(a)If $\mathrm{I}=\{0\}$ or $\mathrm{N}$, then the five properties fuzzy almost strong I -open, fuzzy strong $\beta$-I -open, fuzzy almost-I -open fuzzy $\beta$-I -open and fuzzy $\beta$-open are all equivalent of one another.

(b) If $\mathrm{I}=\rho(\mathrm{X})$, then

(1) the five properties fuzzy open, fuzzy $\alpha$-I -open, fuzzy pre-I -open, fuzzy semi-I open and fuzzy strong $\beta$-I -open are all equivalent of one another.

(2) a subset $A$ of $X$ is fuzzy $\beta$-I -open if and only if $A$ is fuzzy semi-I -open.

\section{Proof :(a)}

(1) Let $\mathrm{I}=\{0\}$. Then $\mathrm{A}^{*}=\mathrm{Cl}(\mathrm{A})$ for any subset of $\mathrm{X}$ and $\mathrm{Cl}^{*}(\mathrm{~A})=\mathrm{AVA} * \mathrm{Cl}(\mathrm{A})$. Therefore, we have $\mathrm{A} \leq$ $\mathrm{Cl}(\operatorname{Int}(\mathrm{Cl}(\mathrm{A})))=\mathrm{Cl}\left(\operatorname{Int}\left(\mathrm{A}^{*}\right)\right)=\mathrm{Cl}^{*}\left(\operatorname{Int}\left(\mathrm{A}^{*}\right)\right)$. This shows that every fuzzy $\beta$-open set is fuzzy almost strong I open.

(2) Let $\mathrm{I}=\mathrm{N}$. Then $\mathrm{A}^{*}=\mathrm{Cl}(\operatorname{Int}(\mathrm{Cl}(\mathrm{A})))$ for any subset $\mathrm{A}$ of $\mathrm{X}$.If $\mathrm{A}$ is fuzzy $\beta$-open, $\mathrm{A} \leq \mathrm{Cl}(\operatorname{Int}(\mathrm{Cl}(\mathrm{A})))$ and $\mathrm{Cl}(\mathrm{A})=\mathrm{Cl}(\operatorname{Int}(\mathrm{Cl}(\mathrm{A})))=\mathrm{A}^{*}$. Therefore, we have $\mathrm{Cl}(\mathrm{A})=\mathrm{A} \vee \mathrm{A}^{*}=\mathrm{Cl}(\mathrm{A})$ for each fuzzy $\beta$-open set $A$ and hence $A \leq \mathrm{Cl}(\operatorname{Int}(\mathrm{Cl}(\mathrm{A})))=\mathrm{Cl}\left(\operatorname{Int}\left(\mathrm{A}^{*}\right)\right)=\mathrm{Cl}^{*}\left(\operatorname{Int}\left(\mathrm{A}^{*}\right)\right)$. This shows that every fuzzy $\beta$-open set is fuzzy almost strong I -open.

(b) Let $\mathrm{I}=\rho(\mathrm{X})$. Then $\mathrm{A}^{*}=\{0\}$ and $\mathrm{Cl}^{*}(\mathrm{~A})=\mathrm{A} \vee \mathrm{A}^{*}=$ Afor every subset $\mathrm{A}$ of $\mathrm{X}$.

(1) If $\mathrm{A}$ is a fuzzy strong $\beta-\mathrm{I}$-open set, then we have $\mathrm{A} \leq \mathrm{Cl}^{*}\left(\operatorname{Int}\left(\mathrm{Cl}^{*}(\mathrm{~A})\right)\right)=\mathrm{Cl}^{*}(\operatorname{Int}(\mathrm{A}))=\operatorname{Int}(\mathrm{A})$. This shows that every fuzzy strong $\beta-\mathrm{I}$-open set is fuzzy open.

(2) If $\mathrm{A}$ is a fuzzy $\beta$-I -open set, then $\mathrm{A} \leq \mathrm{Cl}\left(\operatorname{Int}\left(\mathrm{Cl}^{*}(\mathrm{~A})\right)\right)=\mathrm{Cl}(\operatorname{Int}(\mathrm{A}))$. This shows that every fuzzy $\beta$-I open set is fuzzy semi-open.

Proposition 2.6. For a subset A of a fuzzy ideal topological space $(X, \tau, I)$ the following statements are equivalent.

(a) A is fuzzy almost strong I -open.

(b) $\quad \mathrm{A}$ is fuzzy strong $\beta$-I -open and fuzzy *-dense-in-itself.

Proof :(a) $\Rightarrow$ (b) Every fuzzy almost strong I -open is fuzzy strong $\beta$-I -open from Proposition 2.2. On the other hand, $\mathrm{A} \leq \mathrm{Cl}\left(\operatorname{Int}\left(\mathrm{A}^{*}\right)\right)=\operatorname{Int}\left(\mathrm{A}^{*}\right) \vee\left(\operatorname{Int}\left(\mathrm{A}^{*}\right)\right)^{*} \leq \operatorname{Int}\left(\mathrm{A}^{*}\right) \vee \mathrm{Cl}\left(\operatorname{Int}\left(\mathrm{A}^{*}\right)\right) \leq \mathrm{Cl}\left(\operatorname{Int}\left(\mathrm{A}^{*}\right)\right) \leq \mathrm{Cl}\left(\mathrm{A}^{*}\right)=\mathrm{A}^{*}$ by Lemma 1.1.

(b) $\Rightarrow$ (a) By the assumption, $\mathrm{A} \leq \mathrm{Cl}^{*}\left(\operatorname{Int}\left(\mathrm{CL}^{*}(\mathrm{~A})\right)\right)=\mathrm{Cl}^{*}\left(\operatorname{Int}\left(\mathrm{AVA}^{*}\right)\right) \leq \mathrm{Cl}^{*}\left(\operatorname{Int}\left(\mathrm{A}^{*}\right)\right)$.

This shows that $\mathrm{A} \in \mathrm{FasIO}(\mathrm{X})$.

Remark 2.3. fuzzy strong I -open set and fuzzy *-I -dense in itself are independent of each other as shown in the following example.

Example 2.5. Let $X=\{a, b, c\}$ and $A, B$ be fuzzy subsets of $X$ defined as follows:

$$
\begin{aligned}
& \mathrm{A}(\mathrm{a})=0.2, \mathrm{~A}(\mathrm{~b})=0.3, \mathrm{~A}(\mathrm{c})=0.7 \\
& \mathrm{~B}(\mathrm{a})=0.1, \mathrm{~B}(\mathrm{~b})=0.2, \mathrm{~B}(\mathrm{c})=0.2 .
\end{aligned}
$$

Let $\tau=\{0, B, 1\}$. If we take $I=\rho(X)$, then A is fuzzy $*$-I -dense in itself but A is not fuzzy strong- $\beta$-I -open.

Example 2.6. Let $X=\{a, b, c\}$ and $A, B$ be fuzzy subsets of $X$ defined as follows:

$$
\begin{aligned}
& \mathrm{A}(\mathrm{a})=0.5, \mathrm{~A}(\mathrm{~b})=0.4, \mathrm{~A}(\mathrm{c})=0.6 \\
& \mathrm{~B}(\mathrm{a})=0.5, \mathrm{~B}(\mathrm{~b})=0.5, \mathrm{~B}(\mathrm{c})=0.6
\end{aligned}
$$

Let $\tau=\{0, A, 1\}$. If we take $I=\rho(X)$, then A is fuzzy strong- $\beta$-I -open but A is not fuzzy $*-I$-dense in itself. 


\section{3. $\mathrm{FS}_{\beta \mathrm{I}^{-}}$-set and $\mathrm{FD}_{\mathrm{I}}$-set}

Definition 3.1. A subset of a fuzzy ideal topological space $(X, \tau, I)$ is called

(a) a fuzzy $\mathrm{S}_{\beta} \mathrm{I}-\operatorname{set}$ if $\mathrm{Cl}^{*}\left(\operatorname{Int}\left(\mathrm{Cl}^{*}(\mathrm{~A})\right)\right)=\operatorname{Int}(\mathrm{A})$.

(b) a fuzzy t-I -set[7] if $\operatorname{Int}\left(\mathrm{Cl}^{*}(\mathrm{~A})\right)=\operatorname{Int}(\mathrm{A})$.

Proposition 3.1. Every fuzzy $\mathrm{S}_{\beta} I$-set is a fuzzy t-I -set.

Proof :Let $A$ be a fuzzy $S_{\beta} I-$ set.

Then $\mathrm{Cl}^{*}\left(\operatorname{Int}\left(\mathrm{Cl}^{*}(\mathrm{~A})\right)\right)=\operatorname{Int}(\mathrm{A})$.

SinceInt $(\mathrm{A}) \leq \operatorname{Int}\left(\mathrm{Cl}^{*}(\mathrm{~A})\right)$ and $\operatorname{Int}\left(\mathrm{Cl}^{*}(\mathrm{~A})\right) \leq \mathrm{Cl}^{*}\left(\operatorname{Int}\left(\mathrm{Cl}^{*}(\mathrm{~A})\right)\right)=\operatorname{Int}(\mathrm{A})$. Therefore

$$
\operatorname{Int}(\mathrm{A})=\operatorname{Int}\left(\mathrm{Cl}^{*}(\mathrm{~A})\right) \text { and hence } \mathrm{A} \text { is fuzzy } \mathrm{t}-\mathrm{I} \text {-set. }
$$

Remark 3.1. The converse of Proposition 3.1 need not be true is shown by the following example.

Example 3.1. Let $X=\{a, b, c\}$ and $A, B$ be fuzzy subsets of $X$ defined as follows:

$$
\begin{aligned}
& \mathrm{A}(\mathrm{a})=0.2, \mathrm{~A}(\mathrm{~b})=0.3, \mathrm{~A}(\mathrm{c})=0.7 \\
& \mathrm{~B}(\mathrm{a})=0.1, \mathrm{~B}(\mathrm{~b})=0.2, \mathrm{~B}(\mathrm{c})=0.2 .
\end{aligned}
$$

Let $\tau=\{0, \mathrm{~B}, 1\}$. If we take $\mathrm{I}=\{0\}$, then $\mathrm{A}$ is fuzzy $\mathrm{t}-\mathrm{I}$-set but $\mathrm{A}$ is not fuzzyS $\mathrm{S}_{\beta} \mathrm{I}$-open.

Definition 3.2. A subset $A$ of a fuzzy ideal topological space $(X, \tau, I)$ is called fuzzy $D_{I}-\operatorname{set}\left(\operatorname{resp}\right.$. fuzzy $B_{I}$ set[2]) if $A=U \wedge V$, where $U \in \tau$ and $V$ is fuzzy $S_{\beta} I-\operatorname{set}($ resp. fuzzy $t-I$-set).

We denote the family of all fuzzy $D_{I}$-sets(resp. fuzzy $S_{\beta I}$-sets, fuzzy $B_{I}$-sets) by $F_{I}(X)\left(r e s p . F_{\beta} I(X), F_{I}(X)-\right.$ set).

Proposition 3.2. In a fuzzy ideal topological space $(X, \tau, I), F_{\beta}(X) \leq F_{I}(X), \tau \leq F_{I}(X)$ and $F D_{I}(X) \leq F_{I}(X)$.

Proof: Since $\mathrm{X} \in \tau \wedge \mathrm{FS}_{\beta} \mathrm{I}(\mathrm{X})$ and every $\mathrm{FS}_{\beta} \mathrm{I}$-set is a fuzzy t-I -set by proposition 3.1, the inclusions are obvious.

Proposition 3.3. For a subset A of a fuzzy ideal topological space $(\mathrm{X}, \tau, \mathrm{I})$, the following properties are equivalent:

(a) A is fuzzy open.

(b) $\mathrm{A}$ is fuzzy strong $\beta-\mathrm{I}$-open and fuzzy $\mathrm{D}_{\mathrm{I}}$-set.

\section{Proof :}

(a) $\Rightarrow$ (b) This is obvious.

(b) $\Rightarrow$ (a) Let A be a fuzzy strong $\beta$-I -open and fuzzy $D_{I}$-set. Then

$\mathrm{A} \leq \mathrm{Cl}^{*}\left(\operatorname{Int}\left(\mathrm{Cl}^{*}(\mathrm{~A})\right)\right)=\mathrm{Cl}^{*}\left(\operatorname{Int}\left(\mathrm{Cl}^{*}(\mathrm{U} \wedge \mathrm{V})\right)\right) \leq \mathrm{Cl}^{*}\left(\operatorname{Int}\left(\mathrm{Cl}^{*}(\mathrm{U}) \wedge \mathrm{Cl}^{*}(\mathrm{~V})\right)\right)=$

$\mathrm{Cl}^{*}\left(\operatorname{Int}\left(\mathrm{Cl}^{*}(\mathrm{U})\right) \wedge \operatorname{Int}\left(\mathrm{Cl}^{*}(\mathrm{~V})\right)\right) \leq \mathrm{Cl}^{*}\left(\operatorname{Int}\left(\mathrm{Cl}^{*}(\mathrm{U})\right)\right) \wedge \mathrm{Cl}^{*}\left(\operatorname{Int}\left(\mathrm{Cl}^{*}(\mathrm{~V})\right)\right)=\mathrm{Cl}^{*}\left(\operatorname{Int}\left(\mathrm{Cl}^{*}(\mathrm{U})\right)\right) \wedge$

$\operatorname{Int}(\mathrm{V})$, where $\mathrm{V} \in \mathrm{FS}_{\beta} \mathrm{I}(\mathrm{X})$ and $\mathrm{U} \in \tau$. Hence $\mathrm{A} \leq \mathrm{U}$ and $\operatorname{Int}(\mathrm{A}) \leq \mathrm{A} \leq \mathrm{U} \wedge \operatorname{Int}(\mathrm{V})=\operatorname{Int}(\mathrm{A})$.

Thus $\mathrm{A} \in \tau$. 
Remark 3.2. The following Examples show that fuzzy strong $\beta$-I -open set and fuzzy $D_{I}$-sets are independent notions.

Example 3.2. Let $X=\{a, b, c\}$ and $A, B, C$ be fuzzy subsets of $X$ defined asfollows:

$$
\begin{aligned}
& \mathrm{A}(\mathrm{a})=0.6, \mathrm{~A}(\mathrm{~b})=0.7, \mathrm{~A}(\mathrm{c})=0.5 \\
& \mathrm{~B}(\mathrm{a})=0.4, \mathrm{~B}(\mathrm{~b})=0.3, \mathrm{~B}(\mathrm{c})=0.2 . \\
& \mathrm{C}(\mathrm{a})=0.5, \mathrm{C}(\mathrm{b})=0.7, \mathrm{C}(\mathrm{c})=0.4
\end{aligned}
$$

Let $\tau=\{0, \mathrm{C}, 1\}$. If we take $\mathrm{I}=\{0\}$, then $\mathrm{B}=\mathrm{B} \wedge \mathrm{C}$ is fuzzy $\mathrm{D}_{\mathrm{I}}$-set but $\mathrm{B}$ is notfuzzy strong $\beta-\mathrm{I}$ -open set.

Example 3.3. Let $X=\{a, b, c\}$ and $A, B$ be fuzzy subsets of $X$ defined as follows:

$$
\begin{aligned}
& \mathrm{A}(\mathrm{a})=0.5, \mathrm{~A}(\mathrm{~b})=0.4, \mathrm{~A}(\mathrm{c})=0.6 \\
& \mathrm{~B}(\mathrm{a})=0.5, \mathrm{~B}(\mathrm{~b})=0.5, \mathrm{~B}(\mathrm{c})=0.6
\end{aligned}
$$

Let $\tau=\{0, A, 1\}$. If we take $I=\{0\}$, then $A$ is fuzzy strong- $\beta-I-$ open but $A=A \wedge B$ is not fuzzy $D_{I}$-set.

\section{Decomposition of fuzzy almost strong I-continuity and fuzzy continuity}

Definition 4.1. A function $\mathrm{f}:(\mathrm{X}, \tau, \mathrm{I}) \rightarrow(\mathrm{Y}, \sigma)$ is said to be fuzzy strongly $\beta \mathrm{I}$-continuous(resp. fuzzy $\mathrm{D}_{\mathrm{I}}$ continuous, fuzzy $B_{I}$-continuous) if for every $V \in \sigma, f^{-1}(V)$ is fuzzy strong $\beta$-I -open(resp. fuzzy $D_{I}$-set, fuzzy $\left.B_{I}-\operatorname{set}\right)$ of $(X, \tau, I)$.

Definition 4.2. A function $\mathrm{f}:(\mathrm{X}, \tau, \mathrm{I}) \rightarrow(\mathrm{Y}, \sigma)$ is said to be fuzzy almost strong-I continuous if for every $\mathrm{V} \in \sigma$, $\mathrm{f}^{-1}(\mathrm{~V})$ is fuzzy strong almost-I -open set of $(\mathrm{X}, \tau, \mathrm{I})$.

Theorem 4.1. For a function $\mathrm{f}:(\mathrm{X}, \tau, \mathrm{I}) \rightarrow(\mathrm{Y}, \sigma)$, the following statements areequivalent:

(a) A is fuzzy almost strong I-continuous.

(b) A is fuzzy strong $\beta-\mathrm{I}$-continuous and fuzzy $*-\mathrm{I}$-continuous.

Proof :This follows from Proposition 2.6.

Theorem 4.2. For a function $\mathrm{f}:(\mathrm{X}, \tau, \mathrm{I}) \rightarrow(\mathrm{Y}, \sigma)$, the following statements areequivalent:

(a) A is fuzzy continuous.

(b) $\mathrm{A}$ is fuzzy strong $\beta-\mathrm{I}$-continuous and fuzzy $\mathrm{D}_{\mathrm{I}}$-continuous.

Proof :This follows from Proposition 3.3.

\section{References}

1) E. Hatir, S. Jafari, Fuzzy semi-I-open sets and fuzzy semi-I-continuity via fuzzy idealization, Chaos, Solitons Fractals, 34 (2007), 1220-1224.

2) A. A. Nasef, E.Hatir, On fuzzy pre-I-open sets and a decomposition of fuzzy I continuity, Chaos, Solitons Fractals, 40 (2007), 1185-1189.
3) C. L. Chang, Fuzzy topological spaces, J. Math. Anal. Appl., 24(1968), 182-190.

4) D. Jankovic and T. R. Hamlett, New topologies from old via ideals, Amer. Math. Monthly, 97(1990), 295-310.

5) K. Kuratowski, Topology, Vol. 1 (transl.), Academic press, New York, (1966). 
International Journal of Trend in Scientific Research and Development (IJTSRD) ISSN: 2456-6470

6) R. A. Mahmoud, Fuzzy ideals, fuzzy local function and fuzzy topology, J. Fuzzy Math. Los Angels, 5(1)(1997), 117-123.

7) V. Chitra, K. Malarvizhi, M. Narmadha, Decomposition of weaker forms of continuity via fuzzy ideals, International Journal of Scientific Research and Education, Volume 4, Issue 08, (2016), 5610-5617, ISSN(e):2321-7545

8) D. Sarkar, Fuzzy ideal theory, fuzzy local function and generated fuzzy topology, Fuzzy Sets and Systems, 5(1)(1997), 165-172.
9) R. Vaidyanathaswamy, The localization theory in set topology, Proceedings of the Indian National Science Academy, 20(1945), 51-61.

10) S. Yukesel, E. Gursel and A. Acikgoz, On fuzzy $\alpha-\mathrm{I}$-continuous and fuzzy $\alpha-\mathrm{I}$-open functions, Chaos, Solitons and Fractals, 41(2009), 16911696.

11) L. A. Zadeh, Fuzzy sets, Information and control(Shenyang), 8(1965), 338-353.

12) M. K. Gupta and Rajneesh, Fuzzy $\gamma-\mathrm{I}$-Open sets and a New Decomposition of Fuzzy semi-I continuity via Fuzzy ideals, Int. Journal of Math. Analysis, Vol. 3, 28(2009), 1349-1357. 